behaviour $(\mathrm{p}=0.05)$, internalising problems $(\mathrm{p}=0.02)$ and critical items $(p=0.02)$. BDI scores did not differ between the groups. The VLBW group reported lower mean substance use ( $\mathrm{p}$ $=0.04$ ), mainly due to less use of alcohol. Furthermore, they reported having fewer friends, less closeness to friends, and less time spent with friends compared with controls $(p=0.05)$. When excluding 11 participants with cerebral palsy and/or low intelligence quotient ( $<2 \mathrm{SD}$ of mean in the control group), the scores for critical items, anxious/depressed and substance use were essentially the same (p-values: 0.04-0.07).

Conclusion The VLBW group reported more emotional problems than controls, and also a higher level of clinically relevant psychiatric symptoms. The findings may indicate that anxiety symptoms and a cautious lifestyle with regard to substance use are characteristics of VLBW individuals in young adulthood.

\section{PO-0826 CONOTRUNCAL HEART DEFECT IN A PATIENT WITH CONGENITAL DISORDER OF GLYCOSYLATION TYPE IA}

${ }^{1}$ A Felipe, ${ }^{2} \mathrm{DC}$ Albert, ${ }^{3} \mathrm{M}$ Girós, ${ }^{1} \mathrm{~A}$ Macaya. ${ }^{1}$ Pediatric Neurology, Hospital Universitari Vall d'Hebron, Barcelona, Spain; ${ }^{2}$ Pediatric Cardiology, Hospital Universitari Vall d'Hebron, Barcelona, Spain; ${ }^{3}$ Department of Inborn Errors of Metabolism Biochemistry and Molecular Genetics, Hospital Clínic, Barcelona, Spain

10.1136/archdischild-2014-307384.1459

Background and aims Conotruncal heart defects (CTHD) represent $15-20 \%$ of congenital heart defects; common causes are 22q11 microdelection syndrome and other chromosomal rearrangements. Congenital disorders of glycosylation (CDG) are a group of inherited multisystem disorders caused by defective glycosylation of proteins and lipids. Type I CDG is a group of heterogeneous disorders involving defective synthesis or transfer of a lipid-linked oligosaccharide precursor. The most prevalent cardiac abnormalities are cardiomyopathy and pericardial effusion, although CTHD were recently reported in two patients with CDG Ia. We describe a further case of this unusual clinical presentation.

Case report We report a 10 year-old male with neonatal diagnosis of common arterial trunk, repaired at age 17 days. Postoperative course was complicated by cardiopulmonary arrest and allegedly hypoxic ischaemic encephalopathy. He was referred to the paediatric neurology clinic for evaluation of psychomotor delay and epilepsy. Examination at age $2 y$ revealed delayed language, squint and intense hypotonia. Brain MRI revealed cerebral white matter anomalies and cerebellar atrophy, interpreted as result of his hypoxic-ischaemic event. Array-CGH and FISH for $22 \mathrm{q} 11.2$ deletion were normal. At age $8 \mathrm{y}$ he displayed ataxic gait and dysarthric speech; fat pads and inverted nipples were noted. A repeat MRI showed severe cerebellar atrophy, prompting the suspicion of CDG. Transferrin isoform analysis showed a typical CDG Ia pattern. Fibroblast phosphomannomutase activity and PMM2 mutation screen are ongoing.

Conclusions Although cardiomyopathy and pericarditis are common in CDG Ia, this condition should be suspected in CTHD, particularly when encountering unexpected neurodevelopmental delay.

\section{PO-0827 CONOTRUNCAL HEART DEFECT IN A PATIENT WITH CONGENITAL DISORDER OF GLYCOSYLATION TYPE I}

${ }^{1}$ A Felipe, ${ }^{2} \mathrm{DC}$ Albert, ${ }^{3} \mathrm{M}$ Girós, ${ }^{1} \mathrm{~A}$ Macaya. ${ }^{1}$ Pediatric Neurology, Hospital Universitari Vall d'Hebron, Barcelona, Spain; ${ }^{2}$ Pediatric Cardiology, Hospital Universitari Vall
d'Hebron, Barcelona, Spain; ${ }^{3}$ Department of Inborn Errors of Metabolism Biochemistry and Molecular Genetics, Hospital Clinic, Barcelona, Spain

\subsection{6/archdischild-2014-307384.1460}

Background and aims Conotruncal heart defects (CTHD) represent $15-20 \%$ of congenital heart defects; common causes are 22q11 microdelection syndrome and other chromosomal rearrangements. Congenital disorders of glycosylation (CDG) are a group of inherited multisystem disorders caused by defective glycosylation of proteins and lipids. Type I CDG is a group of heterogeneous disorders involving defective synthesis or transfer of a lipid-linked oligosaccharide precursor. The most prevalent cardiac abnormalities are cardiomyopathy and pericardial effusion, although CTHD were recently reported in two patients with PMM2-CDG, the most frequent CDG I. We describe a further case of this unusual clinical presentation.

Case report We report a 10 year-old male with neonatal diagnosis of common arterial trunk, repaired at age 17 days. Postoperative course was complicated by cardiopulmonary arrest and allegedly hypoxic ischaemic encephalopathy. He was referred to the paediatric neurology clinic for evaluation of psychomotor delay and epilepsy. Examination at age $2 \mathrm{y}$ revealed delayed language, squint and intense hypotonia. Brain MRI revealed cerebral white matter anomalies and cerebellar atrophy, interpreted as result of his hypoxic-ischaemic event. Array-CGH and FISH for $22 \mathrm{q} 11.2$ deletion were normal. At age $8 \mathrm{y}$ he displayed ataxic gait and dysarthric speech; fat pads and inverted nipples were noted. A repeat MRI showed severe cerebellar atrophy, prompting the suspicion of CDG. Transferrin isoforms analysis showed a typical CDG I pattern. Fibroblast phosphomannomutase activity and PMM2 mutation screen are ongoing.

Conclusions Although cardiomyopathy and pericarditis are common in CDG I, this condition should be suspected in CTHD, particularly when encountering unexpected neurodevelopmental delay.

\section{PO-0828 BONE MINERAL DENSITY AND VITAMIN D STATUS IN CHILDREN WITH CEREBRAL PALSY}

${ }^{1}$ AK Finbråten, ${ }^{2} \mathrm{U}$ Syversen, ${ }^{1} \mathrm{~J}$ Skranes, ${ }^{3} \mathrm{GL}$ Andersen, ${ }^{4} \mathrm{R}$ Stevenson, ${ }^{1} \mathrm{~T}$ Vik. ${ }^{1}$ Department of Laboratory Medicine Children's and Women's Health, Norwegian University of Science and Technology, Trondheim, Norway; ${ }^{2}$ Department of Cancer Research and Molecular Medicine, Norwegian University of Science and Technology, Trondheim, Norway; ${ }^{3}$ The Cerebral Palsy Register of Norway, Habilitation Center Vestfold Hospital, Tønsberg, Norway; ${ }^{4}$ Department of Pediatrics, University of Virginia, Charlottesville, USA

\subsection{6/archdischild-2014-307384.1461}

Background and aim Children with cerebral palsy (CP) have increased risk for low bone mineral density (BMD). The aim was to explore the difference in BMD between ambulatory and nonambulatory children with $\mathrm{CP}$ and the relationship between vitamin D status and BMD.

Methods Fifty-one children (age range: 8-18 years; 20 girls) with CP participated and had their BMD measured in the lumbar spine (LS) and the distal femur using dual X-ray absorptiometry. Children with GMFCS level I-III were defined as ambulatory ('walkers') while children with level IV-V were defined as non-ambulatory ('non-walkers'). Serum 25-hydroxyvitamin D (25-OHD) concentrations were measured as an indicator of vitamin D status.

Results Mean BMD z-score was considerably lower at the distal femur than in the LS. Non-walkers had lower mean z-scores (range: -1.7 to -5.4 ) than walkers (range: -0.8 to -1.5 ). Among 